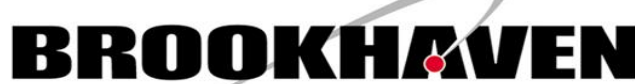 \\ NATIONAL LABORATORY
}

BNL-114798-2017-JA

\section{Controlling Propagation and Coupling of Waveguide Modes Using Phase-Gradient Metasurfaces}

\author{
Z. Li, M. Lu
}

Submitted to Nature Technology

July 2017

Center for Functional Nanomaterials

Brookhaven National Laboratory

\author{
U.S. Department of Energy \\ USDOE Office of Science (SC), \\ Basic Energy Sciences (SC-22)
}

Notice: This manuscript has been authored by employees of Brookhaven Science Associates, LLC under Contract No. DE- SC0012704 with the U.S. Department of Energy. The publisher by accepting the manuscript for publication acknowledges that the United States Government retains a non-exclusive, paid-up, irrevocable, world-wide license to publish or reproduce the published form of this manuscript, or allow others to do so, for United States Government purposes. 


\section{DISCLAIMER}

This report was prepared as an account of work sponsored by an agency of the United States Government. Neither the United States Government nor any agency thereof, nor any of their employees, nor any of their contractors, subcontractors, or their employees, makes any warranty, express or implied, or assumes any legal liability or responsibility for the accuracy, completeness, or any third party's use or the results of such use of any information, apparatus, product, or process disclosed, or represents that its use would not infringe privately owned rights. Reference herein to any specific commercial product, process, or service by trade name, trademark, manufacturer, or otherwise, does not necessarily constitute or imply its endorsement, recommendation, or favoring by the United States Government or any agency thereof or its contractors or subcontractors. The views and opinions of authors expressed herein do not necessarily state or reflect those of the United States Government or any agency thereof. 
Controlling Propagation and Coupling of Waveguide Modes Using Phase-Gradient Metasurfaces

Zhaoyi $\mathrm{Li}^{1}$, Myoung-Hwan $\mathrm{Kim}^{1}{ }^{*}$, Cheng Wang ${ }^{2}$, Zhaohong $\mathrm{Han}^{3}$, Sajan Shrestha ${ }^{1}$, Ming $\mathrm{Lu}^{4}$, Aaron Stein ${ }^{4}$, Anuradha Murthy Agarwal ${ }^{3}$, Marko Lončar ${ }^{2}$, and Nanfang $\mathrm{Yu}^{1}$

${ }^{1}$ Department of Applied Physics and Applied Mathematics, Columbia University, New York, NY 10027, USA

${ }^{2}$ John A. Paulson School of Engineering and Applied Sciences, Harvard University, Cambridge, Massachusetts 02138, USA

${ }^{3}$ Microphotonics Center and Department of Materials Science and Engineering, Massachusetts Institute of Technology, Cambridge, Massachusetts 02139, USA

${ }^{4}$ Center for Functional Nanomaterials, Brookhaven National Laboratory, Upton, NY 11973, USA

* These authors contribute equally to this work

e-mail: anu@mit.edu; loncar@seas.harvard.edu; ny2214@columbia.edu

\begin{abstract}
The research on two-dimensional designer optical structures called "metasurfaces" has mainly focused on controlling the wavefronts of light propagating in free space. The potential of metasurfaces in controlling optical guided waves is underexplored. Here, we show that small-footprint, broadband, and low-loss integrated photonic devices can be created by patterning optical waveguides with gradient metasurface structures consisting of phased arrays of plasmonic or dielectric nano-antennas with subwavelength separations. Optical scattering at subwavelength intervals provides a strong control over guided waves. As a result, metasurface-based integrated photonic devices can have a very compact footprint and broad operation bandwidth. We experimentally demonstrated waveguide mode converters, polarization rotators, and asymmetric optical power transmission in waveguides based on plasmonic gradient metasurfaces. We also experimentally demonstrated all-dielectric polarization rotators based on phased arrays of Mie resonators with negligible insertion losses. The hybrid waveguide-metasurface system represents a new platform for realizing small-footprint, broadband, and loss-loss integrated photonics.
\end{abstract}


The next generation of optical information processing will be based on an integrated platform [14]. The platform is also important for on-chip chemical and biological sensing $[5,6]$. However, major challenges still exist at the device level that prevent reliable integration on a large scale. These challenges include miniaturizing device footprints, increasing device operation bandwidth and robustness, and reducing device insertion losses [1-4]. This paper shows that the integration of optical waveguides and gradient metasurface structures may help address some of these challenges.

The basic concept of metasurfaces is to use arrays of antennas with subwavelength separation and spatially-varying geometric parameters (e.g., antenna shape, size, orientation) to form a spatial distribution of optical response (e.g., phase, amplitude, polarization, optical impedance) that can be used to control the propagation of light at will [7-10]. The focus of investigations so far has been on the physics and application of using metasurfaces to control the wavefronts of light propagating in free space [11-17]. Recently, metasurfaces have emerged as a way to control guided waves and to couple guided waves and waves propagating in free space [18-20].

The phase shift between the scattered light wave from an optical antenna and the incident light wave sweeps a range of $\sim \pi$ across an antenna resonance [7,9]. A gradient metasurface is formed by assembling a linear array of antennas with a constant phase difference $\mathrm{d} \Phi$ and a subwavelength separation $\mathrm{dx}$ between adjacent elements. The gradient metasurface introduces a unidirectional phase gradient $\mathrm{d} \Phi / \mathrm{dx}$, which is equivalent to a unidirectional effective wavevector $\Delta \mathbf{k}$, along the surface, and has been used to demonstrate generalized laws of reflection and refraction [7-12].

A gradient metasurface structure patterned on the top surface of an optical waveguide (Fig. 1) enables asymmetric coupling of waveguide modes. That is, when an incident waveguide mode propagates against $\Delta \mathbf{k}$, the bending angle of its wavevector increases, which corresponds to coupling into higher-order waveguide modes (Fig. 1 upper panel). When an incident waveguide mode propagates along $\Delta \mathbf{k}$, however, due to a continuous increase of wavevector contributed by the gradient metasurface structure, a higher-order waveguide mode is converted to a lower-order one, and the optical power is eventually coupled into a surface wave propagating along the 
plasmonic metasurface (Fig.1 lower panel). The wavevector of the surface wave keeps increasing, and the confinement of the surface wave to the metasurface is enhanced, leading to increasing optical losses. Optical power will thus be dissipated via Joule heating in the plasmonic metasurface. The directional coupling of modes in waveguides integrated with gradient metasurface structures allows us to demonstrate mode conversion, polarization rotation, perfect absorption, and highly asymmetric optical power flow in waveguides.

The gradient metasurfaces are very different from metamaterials and metasurfaces that have been previously used to control guided waves [21-25]. The gradient metasurfaces control guided waves by strong scattering at subwavelength intervals, and the phase jump $\mathrm{d} \Phi$ as a result of optical scattering increases linearly along the metasurface structure. As a result, the gradient metasurface has a strong and collective action on the waveguide modes in the sense that over a propagation distance of only a few times of the wavelength, an effective wavevector of many times of the phase gradient $\mathrm{d} \Phi / \mathrm{dx}$ is imparted onto the incident wavevector, resulting in a large overall wavevector change. This strong and collective effect of the gradient metasurface allows us to substantially reduce the device footprint, and thereby achieve super broadband device performance, which is hard to attain in conventional integrated photonics.

\section{Results}

\section{Asymmetric optical power transmission in waveguides}

Figure 2 shows a device that supports highly asymmetric optical power transmission at $\lambda=2.5$ $\mu \mathrm{m}$ when the fundamental TE waveguide mode is launched along opposite directions. The gradient metasurface structure consists of 56 gold nanorods with different lengths located on the top of a $\mathrm{Si}_{3} \mathrm{~N}_{4}$ waveguide (Fig. 2(a)). Figures 2(b) and (c) are finite-difference time-domain (FDTD) simulations showing mode evolutions in two opposite propagation directions. In the forward direction, the incident $\mathrm{TE}_{00}$ mode is converted into the $\mathrm{TM}_{10}$ mode (Fig. 2(b)). In the backward direction, it couples into surface waves and optical power is strongly absorbed at $\lambda=$ $2.50 \mu \mathrm{m}$ (Fig. 2(c)), with very limited reflection back into the input port or optical scattering into free space and the substrate. The lower panel of Fig. 2(d) shows that optical power flow bends upwards and terminates at the antenna layer on the top surface of the waveguide. The transmission of optical power in the forward and backward directions is $42.9 \%$ and $0.21 \%$, 
respectively, at $\lambda=2.50 \mu \mathrm{m}$ (Fig. 2(e)). The ratio of transmission spectra is maintained at a high level (>100) between $\lambda=2.44 \mu \mathrm{m}$ and $2.64 \mu \mathrm{m}$ and reaches its peak of $\sim 204$ at $\lambda=2.50 \mu \mathrm{m}$ (Fig. 2(e)).

We developed a special coupled-mode theory to model the highly asymmetric mode coupling in the device (Supplementary Section VIII). Figures 2(f) and 2(g) show that our coupled-mode theory can describe the mode evolution in both propagation directions accurately. The most important feature of our theory that distinguishes it from the conventional coupled-mode theory $[26,27]$ is that the coefficients that govern the coupling strength between two modes are different (e.g., $\kappa_{12} \neq \kappa_{21}$ for the coupling between $\mathrm{TE}_{00}$ and $\mathrm{TM}_{10}$ modes and $\kappa_{13} \neq \kappa_{31}$ for the coupling between $\mathrm{TE}_{00}$ and surface waves). The physical reason for the asymmetric coupling coefficients is that the unidirectional phase gradient provided by the metasurface structure breaks the symmetry of optical power transfer between two modes. For example, a phase gradient $\mathrm{d} \Phi / \mathrm{dx}=\Delta \mathbf{k}$ that is aligned with the light propagation direction will facilitate a higher-order waveguide mode to couple into a lower-order one, but will inhibit the reverse process. Note that our devices are reciprocal and do not break time-reversal symmetry; details on this will be provided in the discussion section.

We experimentally demonstrated asymmetric optical power flow in waveguides patterned with gradient metasurfaces. The devices consist of a Y-branch waveguide and two gradient metasurface structures with opposite orientations (Figs. 3(a)-(b)). Each gradient metasurface structure is positioned at the center of the top surface of one waveguide branch and consists of a phased array of gold nanorod antennas according to the design shown in Fig. 2(a). In the experiments, an incident laser beam with tunable wavelength between 2.40 and $2.50 \mu \mathrm{m}$ and controllable polarization was coupled into the input port of the device using a single mode midinfrared fiber, and excited preferentially the $\mathrm{TE}_{00}$ waveguide mode. An infrared camera was used to image the output ports of the two waveguide branches, and the ratio of light transmission through the two ports were monitored as a function of wavelength. Without antenna arrays, the input light is separated equally between the two branches and the two optical spots at the output ports have the same intensity. However, in devices patterned with gradient metasurfaces, we observed large differences in light transmission through the two ports (Figs. 3(c)-(d)) (see also 
Supplementary Section III). Figure 3(c) show images of the two optical spots at the output ports at different wavelengths ranging from 2.43 to $2.47 \mu \mathrm{m}$. In the right branch, light is efficiently converted into surface waves by the gradient metasurface and optical transmission is small. In the left branch, the incident $\mathrm{TE}_{00}$ mode is converted to the $\mathrm{TM}_{10}$ mode, which leads to large optical transmission. The differential transmission reaches its peak value of $\sim 35$ around $\lambda=2.45$ $\mu \mathrm{m}$ (Fig. 3(d)).

\section{Mid-infrared waveguide mode converters based on plasmonic gradient metasurfaces}

Figure 4(a) shows a few waveguide mode converters operating around the mid-infrared wavelength of $4 \mu \mathrm{m}$. The gradient metasurfaces consist of phased arrays of gold nanorod antennas patterned on the top surface of silicon waveguides. The incident TE fundamental waveguide mode is converted into the $\mathrm{TM}_{00}$, $\mathrm{TM}_{10}$, or $\mathrm{TE}_{10}$ mode, depending on the design parameters, which include the phase gradient $\mathrm{d} \Phi / \mathrm{dx}$, array length, offset of the antenna array from the waveguide center, and antenna orientation. The third column in Fig. 4(a) shows FDTD simulations of mode evolution when light propagates from the left to the right through a region of the waveguide patterned with the gradient metasurfaces. The collective action of the antennas can be quantified by the change of wavevector during the mode conversion process. For example, the effective mode index decreases from $\mathrm{n}_{\mathrm{eff}}=2.74$ to 1.78 in the $\mathrm{TE}_{00}$-to- $\mathrm{TM}_{10}$ mode convertor, which corresponds to 7.6 times of the phase gradient $\mathrm{d} \Phi / \mathrm{dx}$, where $\mathrm{d} \Phi=5.7^{\circ}$ and $\mathrm{dx}=500 \mathrm{~nm}$. This large change to the effective mode index occurs during a propagation distance of $12 \mu \mathrm{m}$ (i.e., length of the antenna array), which is just three times of the free space wavelength, $\lambda=4$ $\mu \mathrm{m}$. The power transmission of the mode converters is between $36 \%$ and $71 \%$ and optical losses are mainly due to absorption in gold nano-antennas. The purity of the converted modes can approach unity and high purity is maintained over a broad wavelength range. For example, the purity of the converted $\mathrm{TM}_{10}$ mode is above $90 \%$ in a wide wavelength range from $\lambda=3.5 \mu \mathrm{m}$ to $5.1 \mu \mathrm{m}$ (Fig. 4(b)).

Figure 5 shows the performance of a few waveguide mode converters fabricated according to the designs in Fig. 4(a). We used a grating coupler to couple the output of a $\lambda=4 \mu \mathrm{m}$ quantum cascade laser into the fundamental TE waveguide mode, which then interacts with gradient 
metasurface structures and is converted into the desired output waveguide modes. The converted modes exit from the cleaved facet at the end of the waveguide and radiate into the far-field. To characterize the converted modes, we measured their far-field profiles by raster scanning a single-pixel InSb detector in front of the waveguide output facet. Figure 5(b) shows that the output from the $\mathrm{TE}_{00}$-to- $\mathrm{TM}_{00}$ mode converter (i.e., polarization rotator) has only one far-field lobe with TM polarization and that the TE-polarized far-field is very weak, indicating that the incident $\mathrm{TE}_{00}$ mode has been completely converted into the $\mathrm{TM}_{00}$ mode. The far-field profiles of the $\mathrm{TE}_{00}$-to- $\mathrm{TM}_{10}$ and $\mathrm{TE}_{00}$-to- $\mathrm{TE}_{10}$ mode converters have two lobes but have $\mathrm{TM}$ and $\mathrm{TE}$ polarization, respectively. The residual TE-polarized component in the far-field is negligible for the $\mathrm{TE}_{00}$-to- $\mathrm{TM}_{10}$ mode converter (Fig. 5(d)), which indicates complete mode conversion. The far-field of the $\mathrm{TE}_{00}$-to-TE 10 mode converter (Fig. 5(f)) is non-ideal because of an imperfectly cleaved waveguide output facet.

\section{Low-loss waveguide mode converters based on dielectric gradient metasurfaces}

The concept of gradient metasurfaces can be implemented in any materials systems that support optical resonances. In particular, gradient metasurfaces based on Mie resonators [28-30] allow us to significantly reduce the insertion losses of waveguide mode converters. Figure 6(a) shows a few such all-dielectric mode converters operating around the telecommunications wavelength of $\lambda=1.55 \mu \mathrm{m}$. The devices consist of silicon nanorods with different lengths located on the top surface of $\mathrm{LiNbO}_{3}$ waveguides. We engineered the dipolar Mie resonances in the silicon nanorods so that the incremental phase between adjacent nano-antennas is a constant (Supplementary Section IV).

We experimentally demonstrated broadband waveguide mode converters based on all-dielectric gradient metasurfaces and observed that the device insertion losses are negligible. Figures 6(b)(d) are SEM images of a fabricated telecom $\mathrm{TE}_{00}$-to- $\mathrm{TM}_{00}$ mode converter according to the design shown in Fig. 6(a). We launched the TE fundamental waveguide mode with a tunable wavelength between $1480 \mathrm{~nm}$ and $1580 \mathrm{~nm}$ into the $\mathrm{TE}_{00}$-to- $\mathrm{TM}_{00}$ mode converters and also into bare waveguides of the same geometry (i.e., length and waveguide cross-sectional dimensions) as the mode converters. We observed no obvious difference between the mode converters and the bare waveguides in their transmitted optical power. For example, the measured transmitted 
optical signal by an InGaAs photodetector for the $\mathrm{TE}_{00}$-to- $\mathrm{TM}_{00}$ mode converters averaged over 6 devices is $1.09 \mathrm{~V}$ with a standard deviation of $0.14 \mathrm{~V}$, while the measured transmitted optical signal of 10 bare waveguides has an average of $1.07 \mathrm{~V}$ and a standard deviation of $0.19 \mathrm{~V}$. We further demonstrated that the waveguide mode converters have broadband performance. Figures 6(e) and (f) show typical measured spectra of the TE- and TM-polarized components of a bare waveguide and of a $\mathrm{TE}_{00}$-to- $\mathrm{TM}_{00}$ mode converter, respectively. The measured TE and TM components must be contributed by the $\mathrm{TE}_{00}$ and $\mathrm{TM}_{00}$ waveguide modes, respectively, because the $\mathrm{LiNbO}_{3}$ waveguides are designed to only support the fundamental TE and TM modes. Thus, the spectra in Fig. 6(e) indicate that high quality $\mathrm{TE}_{00}$ mode (i.e., purity $>99 \%$ ) is coupled into bare waveguides and propagates along the entire length of the waveguides (i.e., $2 \mathrm{~mm}$ ); the spectra in Fig. 6(f) indicate that when the dielectric gradient metasurface is patterned on the waveguides, the $\mathrm{TE}_{00}$ mode launched into the device is converted into the high-purity $\mathrm{TM}_{00}$ mode (i.e., purity $96 \%$ averaged over $\lambda=1480-1580 \mathrm{~nm}$ ). These results provide direct evidence that dielectric metasurfaces based on Mie resonators could be used as a low-loss platform for broadband, compact integrated photonic devices.

\section{Discussion}

Directional waveguide mode coupling demonstrated in this work is distinctive from optical isolation. An optical isolator has an asymmetric scattering matrix that relates the incoming and out-going modes at the ports connected to the device, and can only be realized in systems with nonreciprocity [31], such as systems with magnetic materials [32], nonlinearity [33], or temporal refractive index modulations [34]. However, the performance of our waveguide mode converters is reciprocal. For example, if the $\mathrm{TM}_{10}$ mode is launched into the right port of the second device in Fig. 4(a), it will be converted with high efficiency into the $\mathrm{TE}_{00}$ mode exiting from the left port.

The integration of metasurface structures into integrated photonic circuits represents a new platform for broadband and highly efficient control of guided waves. In this new platform, control of waveguide modes is realized by light scattering at closely packed arrays of nanoantennas, where the distance between neighboring antennas is a few times smaller than the wavelength. Optical scattering at subwavelength intervals provides the strongest control over 
guided waves among all device configurations. Therefore, integrated photonic devices based on metasurfaces could have a footprint much smaller than conventional devices based on ridge waveguides, where control of light is through light propagation and coupling via weak evanescent waves. The footprint of metasurface-based integrated photonic devices could also be smaller than that of devices based on photonic crystals, where control of light is through scattering at intervals (i.e., unit cells of the photonic crystals) comparable to the wavelength. The compact footprint of metasurface-based integrated photonic devices could enable a higher level of device integration, and more importantly, a broader device operation wavelength range, because of an inverse relation between the device dimensions and their working bandwidth [35]. In fact, the mode converters we demonstrated can have lengths as short as twice of the free-space wavelength (e.g., the mid-infrared $\mathrm{TE}_{00}$-to- $\mathrm{TE}_{10}$ mode converter and $\mathrm{TE}_{00}$-to- $\mathrm{TM}_{00}$ polarization rotator in Fig. 5) and can maintain high-purity mode conversion over a wavelength range up to $35 \%$ of the central operating wavelength (Fig. 4(b)), which are not achievable in previously demonstrated devices, including those based on photonic crystals [22], directional couplers [36,37], microring resonators [38], multichannel branching waveguides [39,40], Mach-Zehnder interferometers [41], and periodically structured waveguides [42]. For example, in the last work, which reports the experimental realization of a $\mathrm{TE}_{00}$-to- $\mathrm{TE}_{10}$ mode converter, the period, $\Lambda$, of the structured waveguides is chosen so that the reciprocal lattice vector, $2 \pi / \Lambda$, compensates the wavevector mismatching between two waveguide modes; whereas within each period, the waveguide is structured in a complex way to increase the coupling strength between the two modes. Nevertheless, because of the weak interaction between the modes and the structural perturbation to the waveguide, the length of the $\mathrm{TE}_{00}-\mathrm{to}-\mathrm{TE}_{10}$ mode converter realized in Ref. [42] is 14.5 times of the free-space wavelength. Furthermore, because the design is still based on periodic structures (rather than anisotropic metasurfaces that break the symmetry of waveguide mode coupling), the device works only for a single wavelength.

An additional advantage of using metasurfaces to control waveguide modes is that the scattering of light at nano-antennas can generate new polarization components, which enables us to realize conversion between TE and TM modes (Figs. 4-6), whereas it is difficult to create birefringent waveguides and achieve on-chip polarization rotation in conventional device configurations [43]. A comparison between the performance of our devices based on gradient metasurfaces and that 
of waveguide mode converters and polarization rotators based on other design principles is provided in Supplementary Section IX. It is shown that our metasurface-based devices provide the smallest footprints and the largest operation bandwidth among all devices demonstrated so far.

Active control of light on-chip remains challenging in integrated photonics. On-chip modulation has been mainly based on light propagation over long distances in waveguides that have small perturbations to their refractive indices (e.g., as a result of free carrier generation $[44,45]$ ). Recently, there is a trend to use Mie resonators as building blocks of active photonic devices, taking advantage of their intrinsic low losses and localized resonant modes. For example, people experimentally demonstrated ultrafast all-optical switching based on two-photon absorption in amorphous silicon nanodisks [46], and based on Kerr effects in silicon-based metasurfaces supporting Fano resonances [47]. Mie resonators integrated into optical waveguides and the collective effects of arrays of phased resonators, as discussed in this paper, may help amplify the small refractive index changes as a result of free carrier injection or other nonlinear effects, and pave the way for realizing active integrated photonic devices with small foot prints and broad operation bandwidth.

In summary, our results show that waveguides integrated with gradient metasurfaces consisting of phased array antennas provide a versatile platform for realizing broadband, small-footprint, and low-loss integrated photonic devices. The demonstrated functionalities have great potential for device or system applications. For example, waveguide mode conversion is useful in modedivision and polarization-division multiplexing [36-41], which can increase the capacity of communication channels in integrated photonic circuits. The conversion from waveguide modes to surface waves can be used to build broadband integrated perfect absorbers, on-chip biochemical sensors, and small-footprint integrated photodetectors based on internal photoemission of hot electrons [48]. Furthermore, the effective unidirectional wavevector introduced by the gradient metasurfaces can help broadband phase matching in nonlinear optics. Our preliminary study has shown that broadband, efficient second-harmonic generation can be realized in nonlinear waveguides patterned with gradient metasurfaces. 


\section{Methods}

Design of waveguide mode converters

We tailored the "array factor" and "form factor" of the gradient metasurface structure to realize efficient conversion between two waveguide modes. In the perspective of ray optics, the wavevector of each waveguide mode has its characteristic bending angle (Fig. 1). Therefore, the role of the gradient metasurface should be to bend the wavevector of the incident waveguide mode by a proper amount so that the resulting wavevector matches that of a desired output waveguide mode. The incident waveguide mode interacts with the gradient metasurface consecutively, and each interaction bends the wavevector in accord with the magnitude and direction of the effective wavevector $\Delta \mathbf{k}=\mathrm{d} \Phi / \mathrm{dx}$. Therefore, by controlling the "array factor" via properly choosing $\mathrm{d} \Phi / \mathrm{dx}$ and the length of the gradient metasurface, we can ensure that optical power is preferentially coupled into the desired waveguide mode.

The broadband performance of the mode converters is a result of the small device footprint. The optical analog of uncertainty principle prescribes that an object's spatial dimension is inversely proportional to its momentum spread in the k-space [35]. Our gradient metasurface structure has a length equivalent to only a few wavelengths; therefore, it is able to scatter an incident waveguide mode into any directions within a finite angular range that is inversely proportional to the length of the metasurface structure. The efficiency of coupling into a certain output waveguide mode will be high as long as its wavevector is within that finite angular range, and the wavevector of any other waveguide mode is outside of it.

The length of the metasurface structures we choose ranges from 2 to about 10 times of the freespace wavelength. For example, the mid-infrared $\mathrm{TE}_{00}$-to $\mathrm{TM}_{00}$ and the $\mathrm{TE}_{00}$-to-TE 10 mode converters shown in Fig. 5 both have a length of $7.5 \mu \mathrm{m}$, which is about 1.9 times of their central operating wavelength, $\lambda=4 \mu \mathrm{m}$; the telecom $\mathrm{TE}_{00}$-to-TM $\mathrm{TM}_{00}$ mode converter shown in Fig. 6(b) has a length of $20.3 \mu \mathrm{m}$, which is about 13.3 times of its central operating wavelength, $\lambda=1.53$ $\mu \mathrm{m}$. The length of the metasurface structures cannot be much shorter than a few times of the free-space wavelength because if their length is too short, the uncertainty of the unidirectional wavevector they provide will be too large, which will prevent us from achieving pure mode conversion. 
In addition to the "array factor", the selectivity of mode conversion can be enhanced by engineering the scattering pattern of individual antennas (i.e., "form factor"). Physically, the conversion between waveguide modes is mediated by optical scattering at the antennas. Therefore, an essential condition for efficient coupling from one mode to the other is that the antenna near-fields and the waveguide modes have a large spatial overlap on the waveguide cross-section; that is

$$
\frac{\left|\iint \vec{E}_{\text {antenna }}(y, z) \cdot \vec{E}_{w g}^{*}(y, z) d y d z\right|^{2}}{\left(\iint\left|\vec{E}_{\text {antenna }}(y, z)\right|^{2} d y d z\right) \cdot\left(\iint\left|\vec{E}_{w g}(y, z)\right|^{2} d y d z\right)}
$$

should be large. Here $\vec{E}_{\text {antenna }}(y, z)$ represents near-fields around a single antenna, $\vec{E}_{w g}(y, z)$ is the incident or the output waveguide mode, and the integration is over the waveguide transverse crosssection (i.e., yz-plane). As the antenna near-fields contain both y- and z-polarized components, the gradient metasurfaces are able to facilitate coupling between TE- and TM-polarized waveguide modes. By properly choosing the location and orientation of nanorod antennas on the top surface of the waveguide, we are able to control the value of the above integration and thus fine tune the coupling efficiency between two waveguide modes (Supplementary Section VII).

\section{Device fabrication}

The devices were fabricated by a combination of chemical vapor deposition, electron-beam lithography, and reactive ion etching. For the devices supporting asymmetric optical power transmission (Fig. 3), the $\mathrm{Si}_{3} \mathrm{~N}_{4}$ films were deposited onto $\mathrm{SiO}_{2} / \mathrm{Si}$ wafers using low-pressure chemical vapor deposition (LPCVD). For the mid-infrared waveguide mode converters (Fig. 5), the $\mathrm{Si}$ films were deposited onto $\mathrm{SiO}_{2} / \mathrm{Si}$ wafers using plasma-enhanced chemical vapor deposition (PECVD). Electron-beam lithography was used to pattern gold antenna arrays and alignment marks on the $\mathrm{Si}_{3} \mathrm{~N}_{4}$ or Si films. A second electron-beam lithography step was used to create etch masks to define the waveguides. Chromium was used as the etch mask for the $\mathrm{Si}_{3} \mathrm{~N}_{4}$ waveguides. Polymethyl methacrylate (PMMA) electron-beam resist was used as the etch masks for defining Si waveguides. Care was taken to ensure that the antenna arrays and the waveguide etch masks were precisely aligned. Reactive ion etching was then used to etch waveguides. The etch masks were eventually removed. 
The telecom waveguide mode converters (Fig. 6) were processed from lithium niobate on insulator (LNOI) wafers, which consist of lithium niobate thin films bonded on silica substrates. Electron-beam lithography and reactive ion etching were used to first pattern lithium niobate ridge waveguides. An amorphous silicon (a-Si) layer was then deposited over the etched surface using PECVD. A second electron-beam lithography and reactive ion etching step was used to pattern the a-Si layer that is on the top surface of the lithium niobate waveguides into arrays of nano-antennas. The fabricated waveguides and Si nano-antennas were cladded in a silica layer using PECVD. Finally, waveguide facets were diced and polished to ensure reliable and repeatable coupling efficiencies.

Detailed fabrication processes for the three types of devices shown in Figs. 3, 5 and 6 are described in Supplementary Section I.

\section{Device layout and characterization}

The devices that support asymmetric optical power transmission (Fig. 3) consist of a Y-branch waveguide and two gradient metasurface structures with opposite orientations. Each waveguide branch was patterned with one gradient metasurface. Linearly polarized light from a broadly tunable mid-infrared laser was butt-coupled into the input port of the devices. The guided optical power was equally split at the Y-junction and coupled into the TE fundamental waveguide mode in each of the two branches. A mid-infrared camera was used to image the optical spots at the two output ports of each Y-device. The camera images taken with a wire-grid polarizer were processed to quantify the differential optical power transmission through the two waveguide branches.

The mid-infrared waveguide mode converters (Fig. 5) consist of a grating coupler, a waveguide taper, a narrowed segment of waveguide, a 90-degree waveguide turn, a segment of waveguide patterned with a gradient metasurface structure, a second waveguide taper, and a cleaved facet for light out-coupling. Emission from a quantum cascade laser was collimated and focused onto the grating coupler. The narrowed segment of waveguide was used to purify the TE fundamental waveguide mode. The 90-degree turn in the waveguide was used to separate the signal propagating along the waveguide from the optical background guided by the silicon substrate. 
The incident $\mathrm{TE}_{00}$ mode interacts with the gradient metasurface structure and is converted into a desired output waveguide mode, which eventually radiates into the far-field from the cleaved facet at the end of the device. The converted mode was characterized by raster scanning the farfield using a liquid-nitrogen-cooled single-pixel InSb detector. A wire-grid polarizer was placed in front of the detector to characterize the polarization of the output mode.

The telecom waveguide mode converters (Fig. 6) were characterized using a butt coupling setup. Telecom light with tunable wavelengths was coupled into and collected from the polished facets of the lithium niobate waveguides using tapered lensed fibers. A 3-paddle fiber polarization controller was used to ensure that TE-polarized light was launched into the waveguides. The fiber-collected output light was converted into light propagating in the free space, and the TE and TM output polarization components were separated and detected using an InGaAs photodetector.

Detailed information on device layout and characterization is provided in Supplementary Section II.

\section{Acknowledgement}

The work was supported by the Air Force Office of Scientific Research (grant No. FA9550-14-10389 through a Multidisciplinary University Research Initiative program), Defense Advanced Research Projects Agency Young Faculty Award (grant No. D15AP00111), and National Science Foundation (grant No. ECCS-1307948). Funding from the Ministry of Defense, Singapore, and from the Defense Threat Reduction Agency Grant No. HDTRA1-13-1-0001 is gratefully acknowledged. Research was carried out in part at the Center for Functional Nanomaterials, Brookhaven National Laboratory, which is supported by the U.S. Department of Energy, Office of Basic Energy Sciences, under contract no. DE-SC0012704. We acknowledge helpful discussions with Michal Lipson, Richard Osgood Jr., Pao-tai Lin, and Lin Zhang.

\section{References}

1. Soref, R. The past, present, and future of silicon photonics. IEEE J. Sel. Top. Quantum Electron. 12, 1678 (2006).

2. Soref, R. Mid-infrared photonics in silicon and germanium. Nature Photon. 4, 495-497 (2010). 
3. Wada, K., Luan, H.-C., Lim, D. R. \& Kimerling, L. C. On-chip interconnection beyond semiconductor roadmap: silicon microphotonics. Proc. SPIE 4870, 437 (2002).

4. Jalali, B. \& Fathpour, S. Silicon photonics. IEEE J. Lightwave Technol. 24, 4600-4615 (2006).

5. Soref, R. A., Emelett, S. J. \& Buchwald, W. R. Silicon waveguided components for the longwave infrared region. J. Opt. A: Pure Appl. Opt. 8, 840-848 (2006).

6. Lin, P. T. et al. Low-stress silicon nitride platform for mid-infrared broadband and monolithically integrated microphotonics. Adv. Opt. Mater. 1, 732-739 (2013).

7. Yu, N. \& Capasso, F. Flat optics with designer metasurfaces. Nature Mater. 13, 139-150 (2014).

8. Kildishev, A. V., Boltasseva, A. \& Shalaev, V. M. Planar photonics with metasurfaces. Science 339, 1232009 (2013).

9. Yu, N. et al. Flat optics: Controlling wavefronts with optical antenna metasurfaces. IEEE J. Sel. Top. Quantum Electron. 19, 4700423 (2013).

10. Chen, H.-T., Taylor, A. J. \& Yu, N. A review of metasurfaces: physics and applications. Rep. Prog. Phys. 79, 076401 (2016).

11. Shitrit, N., Bretner, I., Gorodetski, Y., Kleiner, V. \& Hasman, E. Optical spin Hall effects in plasmonic chains. Nano Lett. 11, 2038-2042 (2011).

12. Yu, N. et al. Light propagation with phase discontinuities: generalized laws of reflection and refraction. Science 334, 333-337 (2011).

13. Chen, X. et al. Dual-polarity plasmonic metalens for visible light. Nature Commun. 3, 1198 (2012).

14. Pors, A., Nielsen, M. G., Eriksen, R. L. \& Bozhevolnyi, S. I. Broadband focusing flat mirrors based on plasmonic gradient metasurfaces. Nano Lett. 13, 829-834 (2013).

15. Yang, Y. et al. Dielectric meta-reflectarray for broadband linear polarization conversion and optical vortex generation. Nano Lett. 14, 1394-1399 (2014).

16. Zheng, G. et al. Metasurface holograms reaching 80\% efficiency. Nature Nanotech. 10, 308312 (2015).

17. Wang, Q. et al. Optically reconfigurable metasurfaces and photonic devices based on phase change materials. Nature Photon. 10, 60-65 (2016).

18. Sun, S. et al. Gradient-index meta-surfaces as a bridge linking propagating waves and surface waves. Nature Mater. 11, 426-431 (2012). 
19. Vakil, A. \& Engheta, N. Transformation optics using graphene. Science 332, 1291-1294 (2011).

20. Gok, G. \& Grbic, A. Tailoring the phase and power flow of electromagnetic fields. Phys. Rev. Lett. 111, 233904 (2013).

21. Feng, L. et al. Experimental demonstration of a unidirectional reflectionless parity-time metamaterial at optical frequencies. Nature Mater. 12, 108-113 (2013).

22. Liu, V., Miller, D. A. B. \& Fan, S. Ultra-compact photonic crystal waveguide spatial mode converter and its connection to the optical diode effect. Opt. Express 20, 28388-28397 (2012).

23. Piggott, A. Y. et al. Inverse design and demonstration of a compact and broadband on-chip wavelength demultiplexer. Nature Photon. 9, 374-377 (2015).

24. Shen, B., Wang, P., Polson R. \& Menon, R. An integrated-nanophotonics polarization beamsplitter with $2.4 \times 2.4 \mu \mathrm{m}^{2}$ footprint. Nature Photon. 9, 378-382 (2015).

25. Arango, F. B., Thijssen, R., Brenny, B., Coenen T. \& Koenderink, A. F. Sci. Rep. 5, 10911 (2015).

26. Yariv, A. Optical Electronics in Modern Communications ( $5^{\text {th }}$ edition) (Oxford University Press, 1997).

27. Marcuse, D. Theory of Dielectric Optical Waveguides ( ${ }^{\text {nd }}$ edition) (Academic Press Inc, 1991).

28. Aieta, F., Kats, M. A., Genevet, P. \& Capasso, F. Multiwavelength achromatic metasurfaces by dispersive phase compensation. Science 347, 1342-1345 (2015).

29. Lin, D. M., Fan, P. Y., Hasman, E. \& Brongersma, M. L. Dielectric gradient metasurface optical elements. Science 345, 298-302 (2014).

30. Arbabi, A., Horie, Y., Bagheri, M. \& Faraon, A. Dielectric metasurfaces for complete control of phase and polarization with subwavelength spatial resolution and high transmission. Nature Nanotech. 10, 937-943 (2015).

31. Jalas, D. et al. What is — and what is not — an optical isolator. Nature Photon. 7, 579-582 (2013).

32. Bi, L. et al. On-chip optical isolation in monolithically integrated non-reciprocal optical resonators. Nature Photon. 5, 758-762 (2011).

33. Fan, L. An all-silicon passive optical diode. Science 335, 447-450 (2012). 
34. Lira, H., Yu, Z., Fan, S. \& Lipson, M. Electrically driven nonreciprocity induced by interband photonic transition on a silicon chip. Phys. Rev. Lett. 109, 033901 (2012).

35. Mansuripur, M. Opt. \& Photon. News 13, 44-48 (2002).

36. Ding, Y. et al. On-chip two-mode division multiplexing using tapered directional couplerbased mode multiplexer and demultiplexer. Opt. Express 21, 10376-10382 (2013).

37. Dai, D., Wang, J. \& Shi, Y. Silicon mode (de)multiplexer enabling high capacity photonic networks-on-chip with a single-wavelength-carrier light. Opt. Lett. 38, 1422-1424 (2013).

38. Luo, L.-W. et al. WDM-compatible mode-division multiplexing on a silicon chip. Nature Commun. 5, 3069 (2014).

39. Lee, B.-T. \& Shin, S.-Y. Mode-order converter in a multimode waveguide. Opt. Lett. 28, 1660-1662 (2003).

40. Driscoll, J. B. et al., Asymmetric Y junctions in silicon waveguides for on-chip modedivision multiplexing. Opt. Lett. 38, 1854-1856 (2013).

41. Huang, Y., Xu, G. \& Ho, S.-T. An ultracompact optical mode order converter. IEEE Photon. Technol. Lett. 18, 2281-2283 (2006).

42. Ohana, D., Desiatov, B., Mazurski, N. \& Levy, U. Dielectric metasurface as a platform for spatial mode conversion in nanoscale waveguides, DOI: 10.1021/acs.nanolett.6b04264

43. Velasco, A. V. et al. Ultracompact polarization converter with a dual subwavelength trench built in a silicon-on-insulator waveguide. Opt. Lett. 37, 365-367 (2012).

44. Almeida, V. R., Barrios, C. A. Panepucci, R. R. \& Lipson, M. All-optical control of light on a silicon chip. Nature 431, 1081-1084 (2004).

45. Xu, Q., Schmidt, B., Pradhan, S. \& Lipson, M. Micrometre-scale silicon electro-optic modulator. Nature 435, 325-327 (2005).

46. Shcherbakov, M. R. et al. Ultrafast all-optical switching with magnetic resonances in nonlinear dielectric nanostructures. Nano Lett. 15, 6985-6990 (2015).

47. Yang, Y. et al. Nonlinear Fano-resonant dielectric metasurfaces. Nano Lett. 15, 7388-7393 (2015).

48. Knight, M. W., Sobhani, H., Nordlander, P. \& Halas, N. J. Photodetection with active Optical antennas. Science 332, 702-704 (2011). 


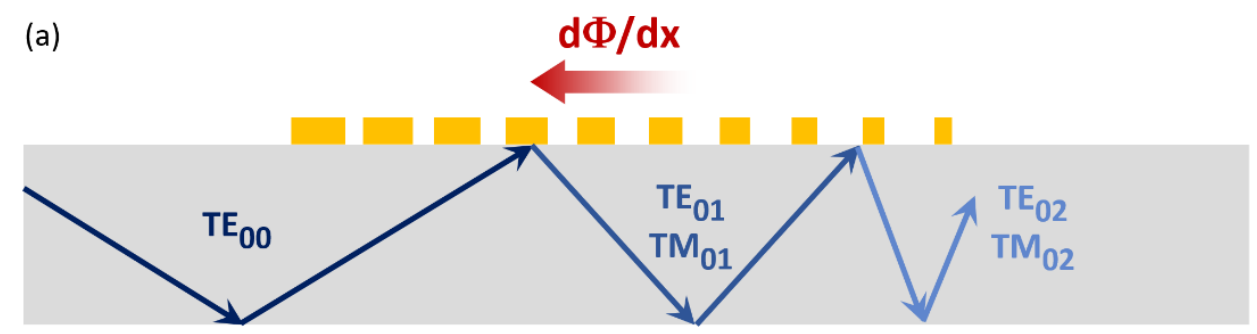

(b)

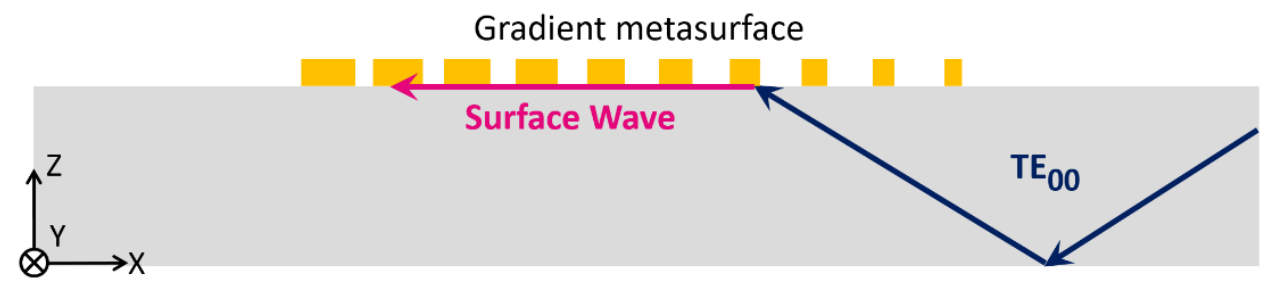

Fig. 1 Ray diagrams to illustrate that a unidirectional phase gradient $\mathrm{d} \Phi / \mathrm{dx}$ introduced by a gradient metasurface causes asymmetric mode coupling in an optical waveguide. In the forward propagation direction, an incident fundamental waveguide mode can be coupling into either higher-order TE or TM modes. In the backward direction, an incident fundamental waveguide mode is coupled into surface waves propagating along the gradient metasurface and optical power is completely absorbed. Here, TE or transverse electric modes are defined as waveguide modes that have their electric field component preferentially in the y-direction, and TM or transverse magnetic modes have their electric field component preferentially in the zdirection [26,27]. The TE(M) mn mode has $(m+1)$ and $(n+1)$ lobes in the $y$ - and $z$-direction, respectively. 

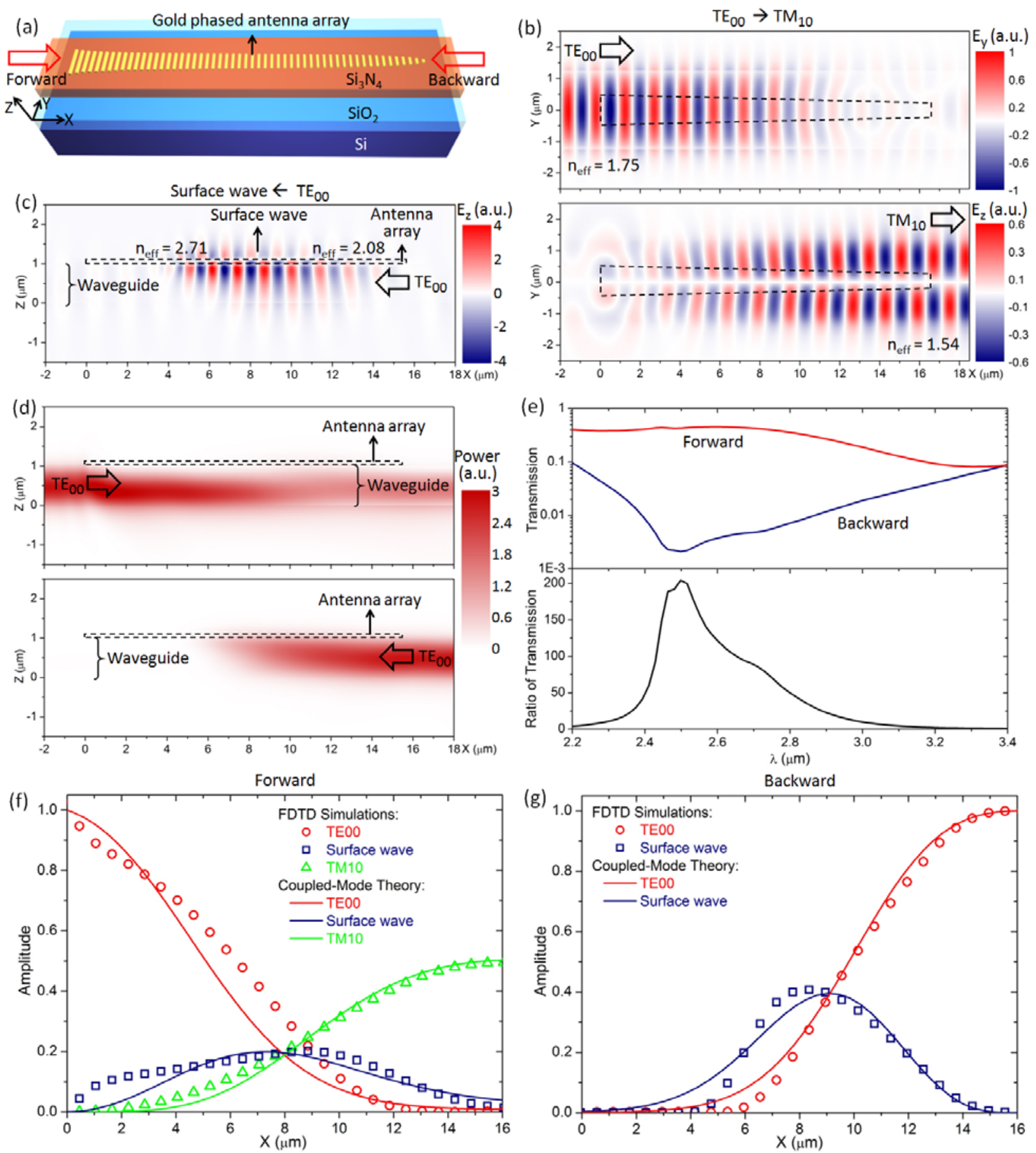

Fig. 2 Asymmetric optical power transmission in waveguides patterned with gradient metasurface structures. (a) Schematic of a device working around $\lambda=2.5 \mu \mathrm{m}$. The $\mathrm{Si}_{3} \mathrm{~N}_{4}$ waveguide is $2.5 \mu \mathrm{m}$ in width and $1.0 \mu \mathrm{m}$ in thickness. The center-to-center distance between adjacent antennas is $300 \mathrm{~nm}$. The length of the antenna array is $16.5 \mu \mathrm{m}$. The detailed design parameters are provided in Supplementary Section V. (b)-(c) Full-wave simulations showing 
conversion of the incident $\mathrm{TE}$ fundamental mode into $\mathrm{TM}_{10}$ mode in the forward propagation direction and into surface waves in the backward direction. The black dashed lines indicate the location of the antenna array. Notice that effective mode index $n_{\text {eff }}$ evolves differently in two opposite propagation directions. (d) Spatial distribution of the magnitude of the Poynting vector, showing highly asymmetric optical power flow as a result of the directional mode conversion. The black dashed lines indicate the location of the antenna array. (e) Optical power transmission spectra of the device in opposite propagation directions and the ratio of the spectra, showing that highly asymmetric optical power transmission is maintained over a broad wavelength range. (f)(g) Mode evolution as a function of propagation distance in opposite propagation directions. Results of coupled-mode theory (curves) have good quantitative agreement with results of fullwave simulations (symbols).

(a)

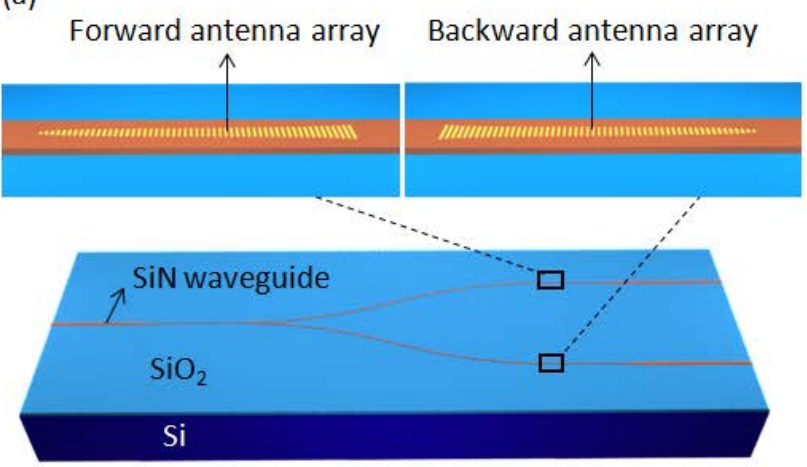

(c)

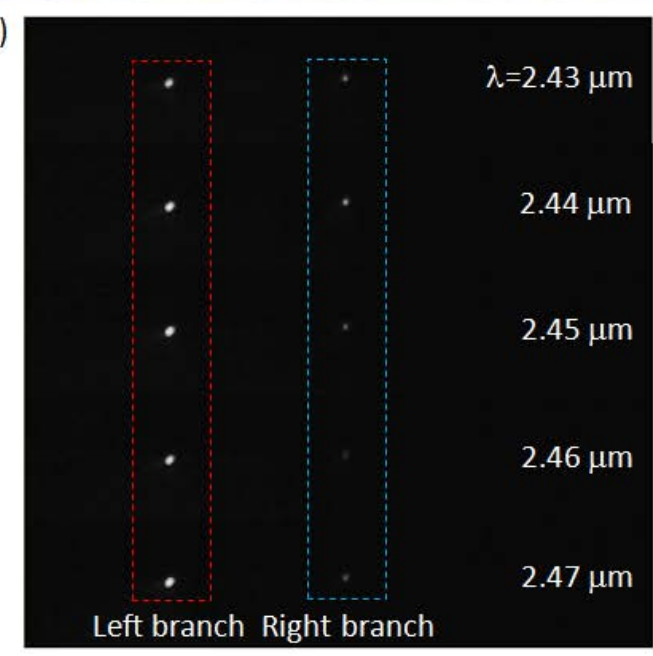

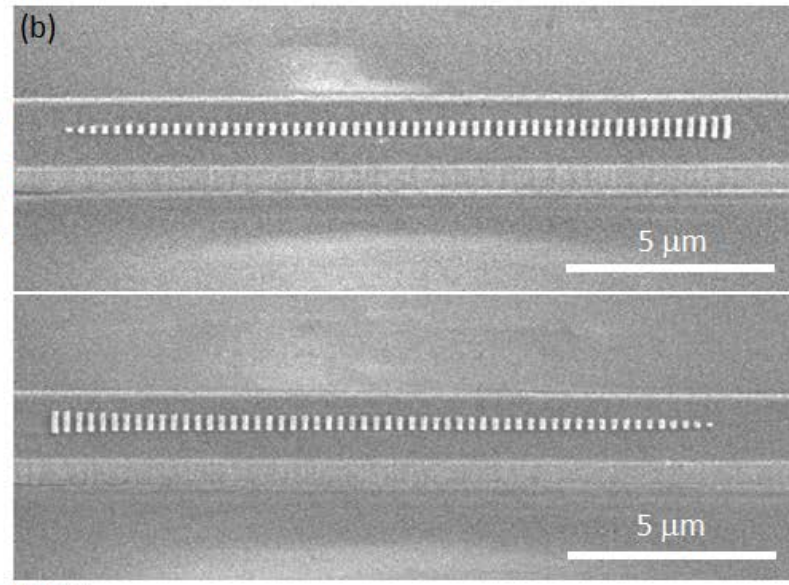

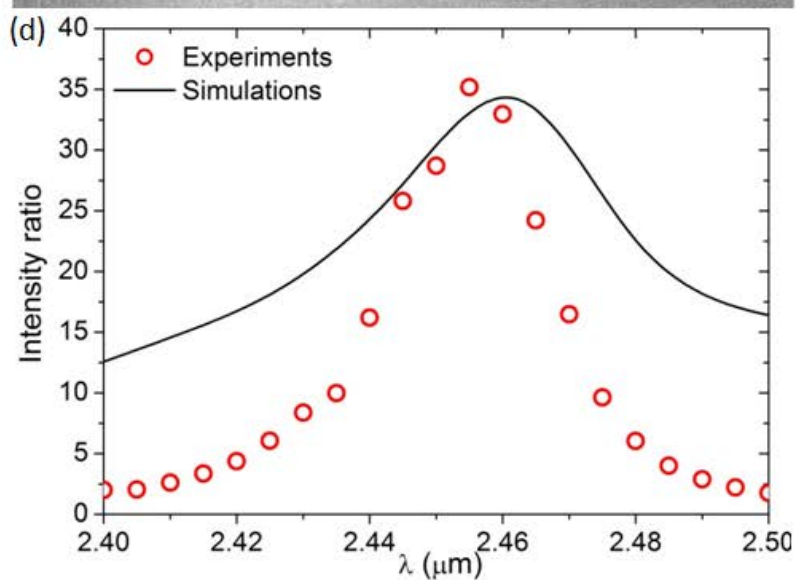

Fig. 3 Experimental demonstration of asymmetric optical power transmission in waveguides patterned with gradient metasurface structures. (a) Schematic of fabricated devices consisting of a Y-branch waveguide and two gradient metasurface structures patterned 
on the waveguide branches. Light is coupled into the device from the left port as the $\mathrm{TE}_{00}$ mode, equally splitted into the two branches still as the $\mathrm{TE}_{00}$ mode, and interacts with the two gradient metasurfaces differently. Insets: Zoom-in view of the device. (b) SEM images of two gradient metasurface structures patterned on the top surfaces of the two $\mathrm{Si}_{3} \mathrm{~N}_{4}$ waveguide branches of a device. The metasurface structures are arranged in two opposite orientations and each consists of 56 gold nanorod antennas with different lengths. (c) Infrared camera images of two output ports of a device at different wavelengths ranging from $2.43 \mu \mathrm{m}$ to $2.47 \mu \mathrm{m}$, showing highly asymmetric power transmission through the two waveguide branches. A polarizer was put in front of the camera to select the TM-polarized component. (d) Measured and simulated ratio of unpolarized peak light intensity at the two output ports compared with simulations. The fabricated devices have slightly under-etched waveguides, which leads to a smaller wavelength range for observing asymmetric optical power flow. 


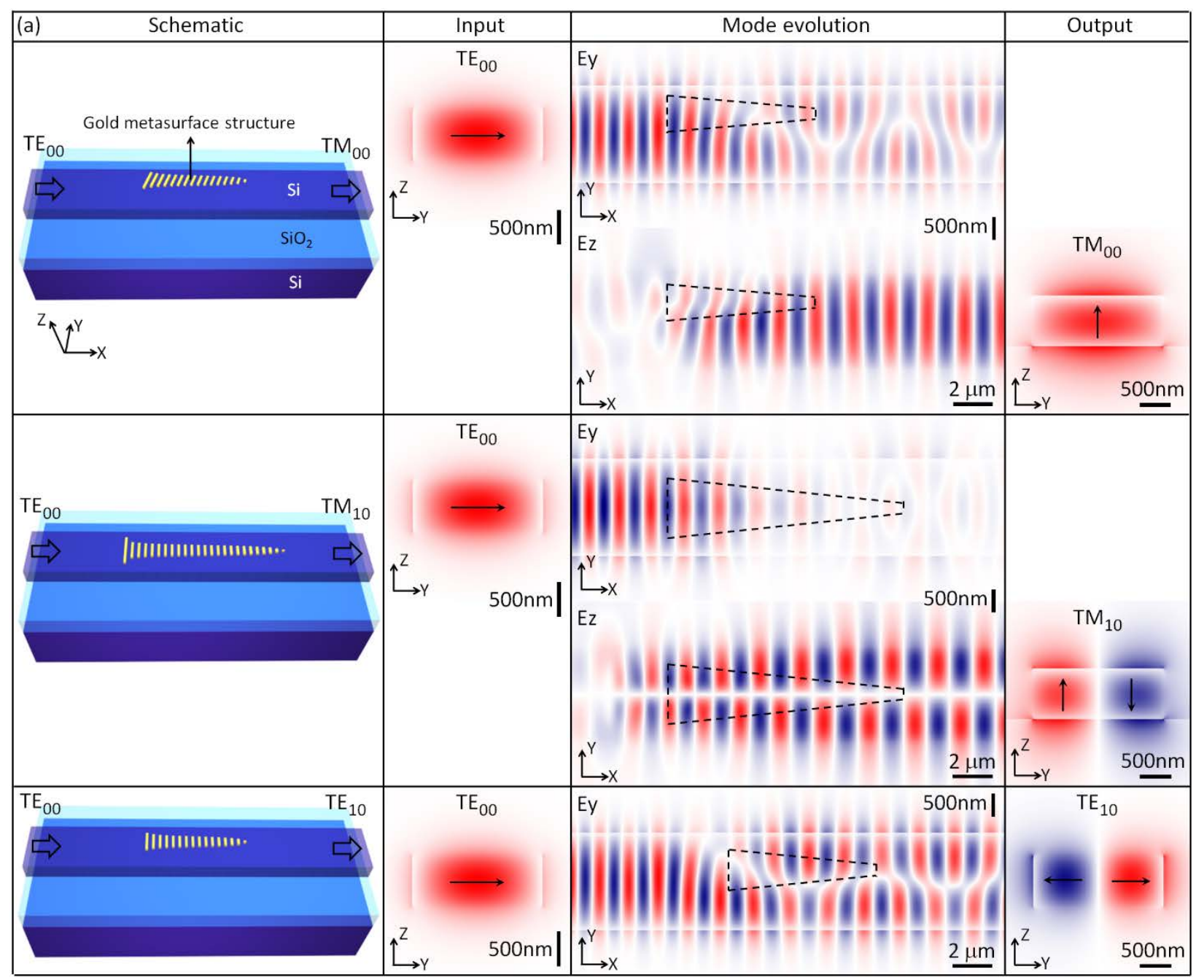

(b)

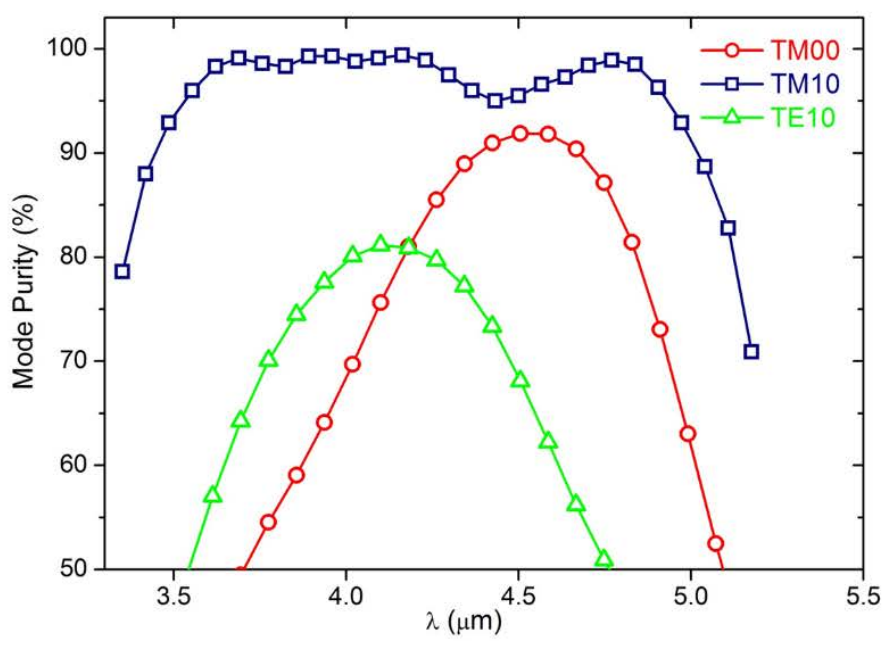

Fig. 4 Mid-infrared waveguide mode converters and polarization rotator based on plasmonic gradient metasurfaces. (a) Simulated device performance. The first column shows device schematics. The gradient metasurface structures consist of gold phased array antennas 
(yellow rods) patterned on silicon waveguides and operate around $\lambda=4 \mu \mathrm{m}$. The silicon waveguides in all devices are $2.1 \mu \mathrm{m}$ in width and $0.75 \mu \mathrm{m}$ in thickness. The thickness of the underlying $\mathrm{SiO}_{2}$ layer is $5 \mu \mathrm{m}$. The lengths of the $\mathrm{TE}_{00}$-to- $\mathrm{TM}_{00}, \mathrm{TE}_{00}$-to- $\mathrm{TM}_{10}$, and $\mathrm{TE}_{00}-\mathrm{to}_{0}-\mathrm{TE}_{10}$ mode converters are, respectively, $7.5 \mu \mathrm{m}, 12 \mu \mathrm{m}$, and $7.5 \mu \mathrm{m}$. Detailed design parameters of the devices are provided in Supplementary Section V. The second and fourth columns show, respectively, waveguide modes at the input and output ports of the devices. The polarization of the electric field component of the modes is indicated by arrows. The third column shows mode evolutions as light propagates from left to right. The peak transmission efficiencies of the three devices from top to bottom are, respectively, 65\%, 36\%, and 71\%. (b) Purity of the converted modes as a function of wavelength, showing that the devices can operate over a broad wavelength range.

(a)

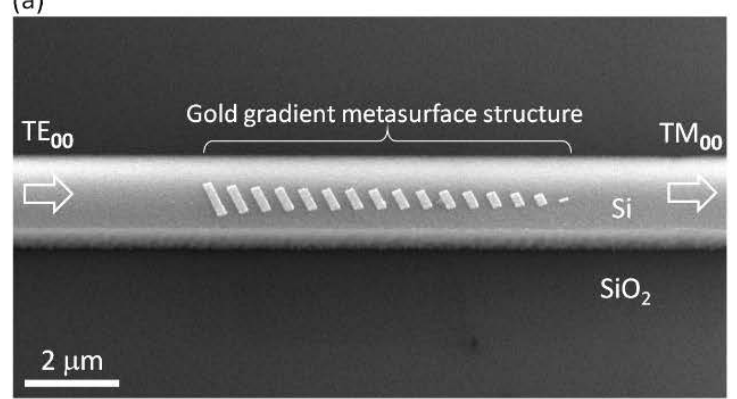

(c)

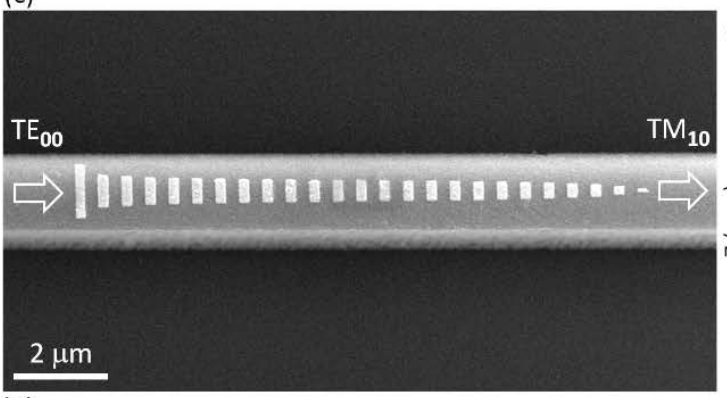

(e)

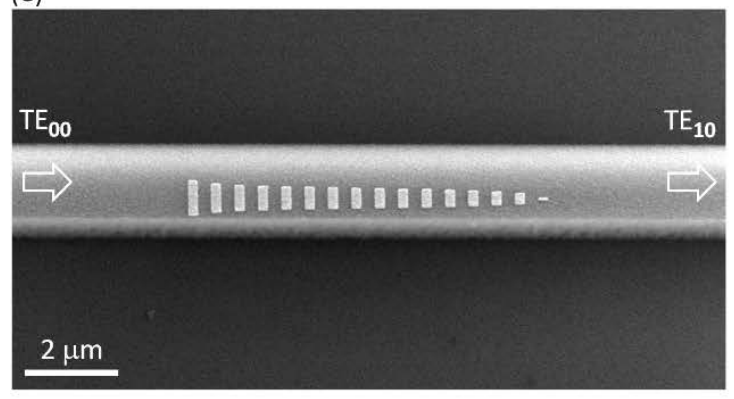

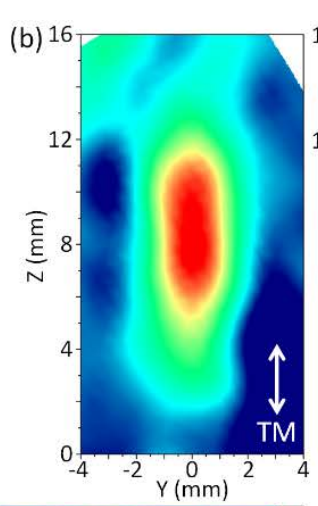
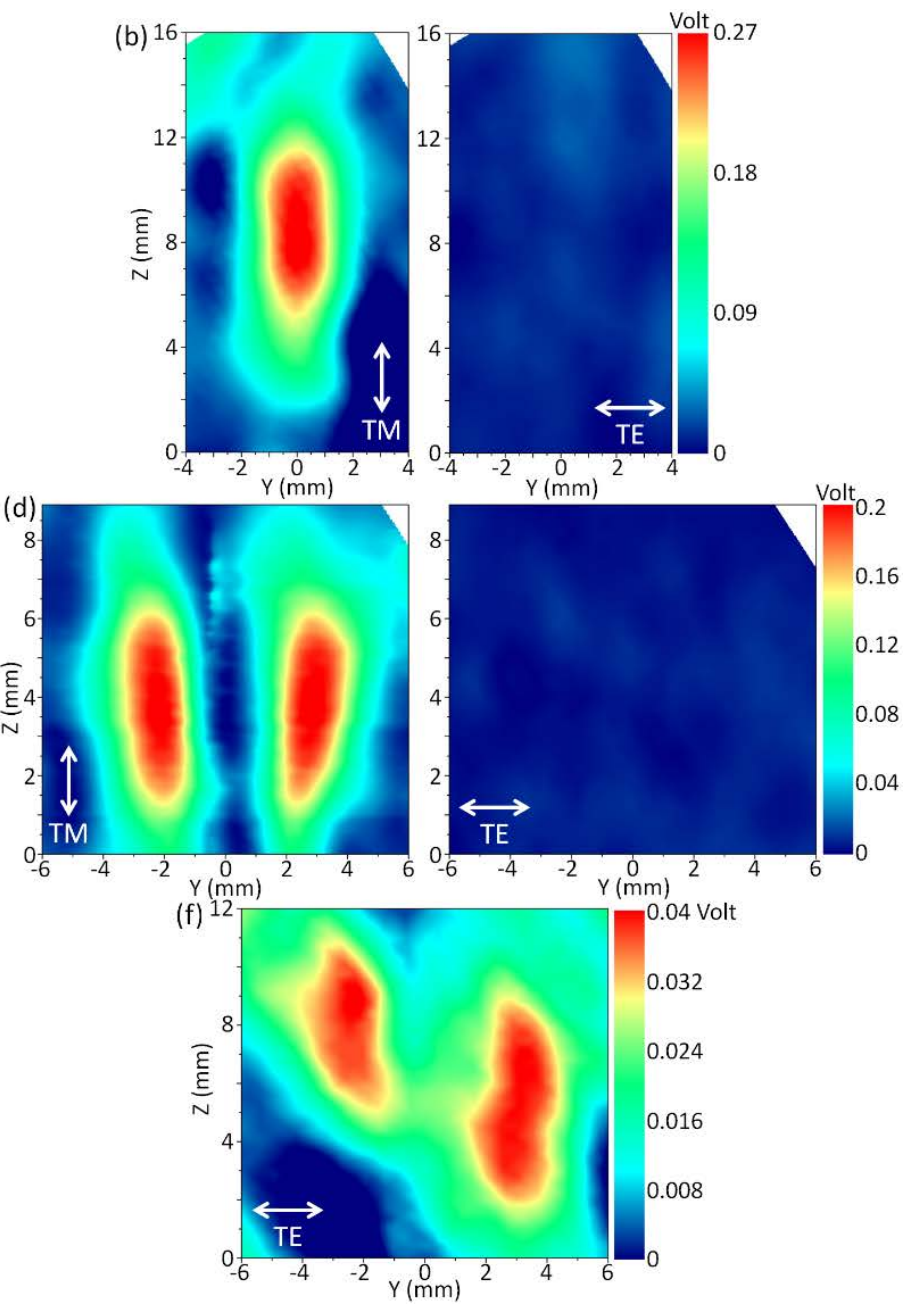
Fig. 5 Experimental demonstration of mid-infrared waveguide mode converters. (a) SEM image of the $\mathrm{TE}_{00}$-to- $\mathrm{TM}_{00}$ mode converter (perspective view, 30 degrees from normal to the waveguide top surface). (b) Measured far-field emission patterns of the $\mathrm{TE}_{00}$-to- $\mathrm{TM}_{00}$ mode converter (i.e., polarization rotator). TM-polarized far-field shows a single lobe in the far field and TE-polarized far-field is very weak. (c) SEM image of the $\mathrm{TE}_{00}$-to-TM 10 mode converter. (d) Measured far-field emission patterns of the $\mathrm{TE}_{00}$-to- $\mathrm{TM}_{10}$ mode converter, showing two lobes in the far field with TM polarization and weak far field with TE-polarized. (e) SEM image of the $\mathrm{TE}_{00}$-to- $\mathrm{TE}_{10}$ mode converter. (f) Measured far-field emission pattern of the $\mathrm{TE}_{00}$-to-TE 10 mode converter. The asymmetric far-field is due to defects on the cleaved waveguide output facet. The weak residual TE-polarized signal in the far field of the $\mathrm{TE}_{00}$-to- $\mathrm{TM}_{00}$ and $\mathrm{TE}_{00}$-to- $\mathrm{TM}_{10}$ mode converters indicates that the conversion from the TE-polarized incident mode to the TMpolarized output modes is quite complete. Notice that the offset of antenna array from the waveguide central axis is chosen to be $200 \mathrm{~nm}$ in (a) and $400 \mathrm{~nm}$ in (e) to achieve high purity mode conversion. 

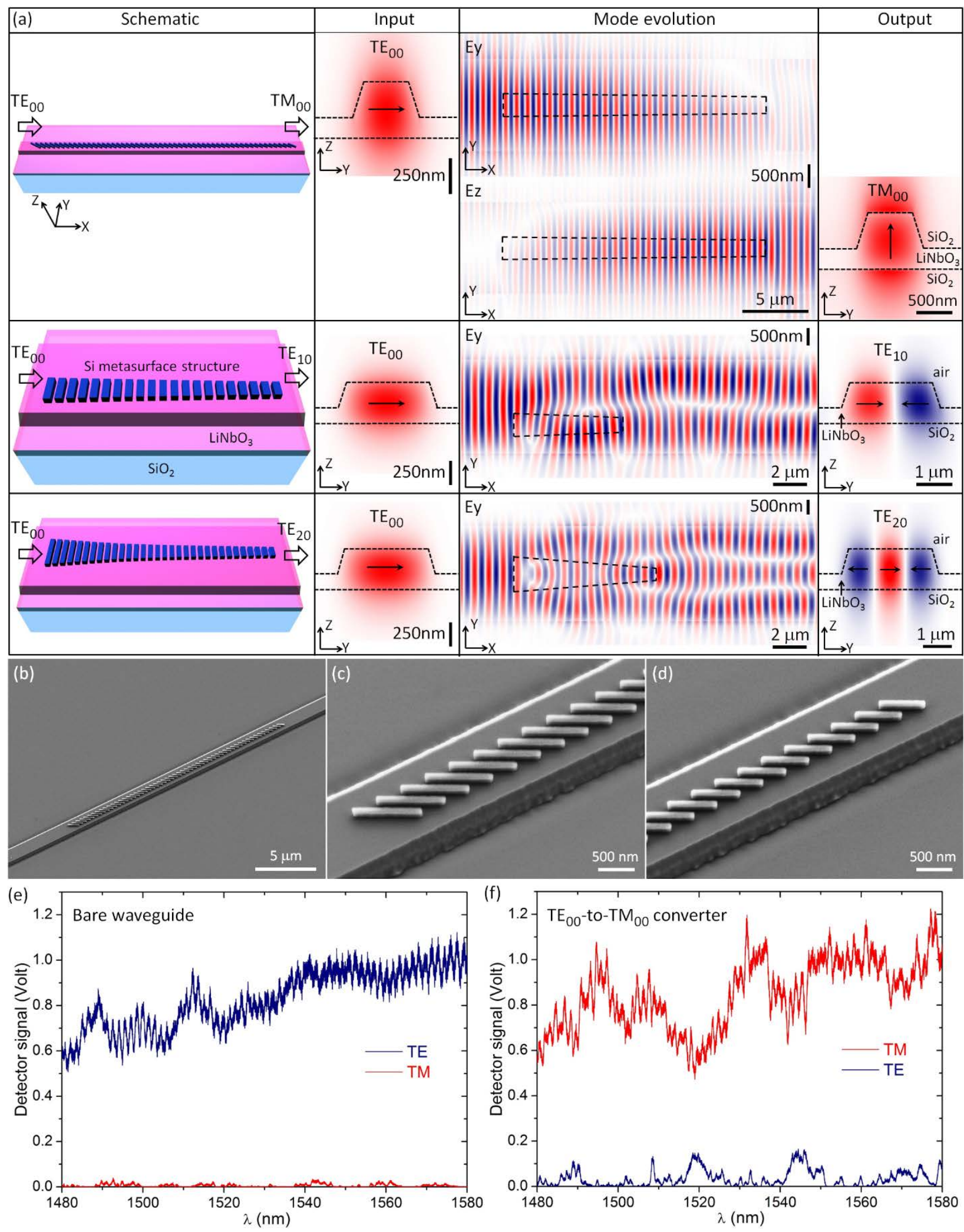

Fig. 6 Telecom waveguide mode converters based on dielectric gradient metasurfaces. (a) Simulated device performance. The first column shows schematics of three waveguide mode 
converters. The gradient metasurface structures consist of phased arrays of silicon antennas (dark blue rods) patterned on $\mathrm{LiNbO}_{3}$ waveguides. The silicon nanorods support dipolar Mie resonances, and are designed to introduce a unidirectional phase gradient along the waveguide. The lengths of the $\mathrm{TE}_{00}$-to- $\mathrm{TM}_{00}, \mathrm{TE}_{00}$-to- $\mathrm{TE}_{10}$, and $\mathrm{TE}_{00}$-to- $\mathrm{TE}_{20}$ mode converters are, respectively, $20.3 \mu \mathrm{m}, 5.4 \mu \mathrm{m}$, and $8.4 \mu \mathrm{m}$. Detailed design parameters of the devices are provided in Supplementary Section V. The second and fourth columns show, respectively, waveguide modes at the input and output ports of the devices. The polarization of the electric field component of the modes is indicated by arrows, and the boundaries of the $\mathrm{LiNbO}_{3}$ waveguide are indicated by dashed lines. The third column shows mode evolutions as light propagates from left to right. The calculated transmission efficiencies of the three devices from top to bottom averaged over $\lambda=1480-1580 \mathrm{~nm}$ are $94.4 \%, 92.9 \%$, and $82.9 \%$, respectively (Supplementary Section III). (b) SEM image of a fabricated $\mathrm{TE}_{00}$-to- $\mathrm{TM}_{00}$ mode converter (before applying an overcoating of $\mathrm{SiO}_{2}$ ). (c) and (d) Zoom-in views of (b). (e) Measured TE and TM polarized components at the output port of a bare waveguide that has the same geometries (i.e., length and cross-sectional dimensions) as the $\mathrm{TE}_{00}$-to- $\mathrm{TM}_{00}$ mode converters. (f) Measured $\mathrm{TE}$ and TM polarized components at the output port of a $\mathrm{TE}_{00}-\mathrm{to}-\mathrm{TM}_{00}$ mode converter, showing that the device operates over a broad wavelength range and has similar power transmission compared to the bare waveguide. 